\title{
Searching for charged lepton flavor violation with the CMS detector
}

\section{Diego Beghin*t}

Universite libre de Bruxelles (ULB)

E-mail: diego.beghin@gmail.com

The CMS Collaboration has performed multiple searches for charged lepton flavor violating (LFV) processes. This talk will be a survey of the latest results of analyses looking for charged LFV decays of known Standard Model particles, and for charged LFV decays of hypothetical new high-energy particles or quantum black holes. No signs of new physics have been found, the current upper limits obtained by CMS on charged LFV phenomena are presented.

The 20th International Workshop on Neutrinos (NuFact2018)

12-18 August 2018

Blacksburg, Virginia

* Speaker.

${ }^{\dagger}$ On behalf of the CMS Collaboration 


\section{Introduction}

Neutrino oscillations were observed for the first time at the Super-Kamiokande detector [1], and have since been confirmed by many other experiments. This raises the question of charged LFV phenomena: do they exist? A straightforward extension of the SM giving Dirac masses for the neutrinos in a similar way that masses are given to other SM fermions could explain the neutrino oscillations, but predicts unobservably small rates for charged LFV phenomena. On the other hand, new physics models such as R-Parity-Violating Supersymmetry (RPV SUSY), see-saw neutrino models, or models with extra dimensions, predict much larger rates for the same charged LFV phenomena. Searches for charged LFV are thus very sensitive to new physics.

CMS is a general purpose particle physics detector located at one of the interaction points of CERN's Large Hadron Collider (LHC) [2]. Many charged LFV searches have been performed there over the years.

\section{2. $\mathbf{Z} \rightarrow \mathrm{e} \mu$ search}

A search of $Z \rightarrow e \mu$ events was performed on $19.7 \mathrm{fb}^{-1}$ worth of data collected in 2012 [3]. A 95\% confidence-level (CL) limit was observed on the branching ratio: $B(\mathrm{Z} \rightarrow \mathrm{e} \mu)<7.3 \cdot 10^{-7}$ (expected: $B<6.7 \cdot 10^{-7}$ ). Indirect low-energy experiments find much stronger constraints of order $5 \cdot 10^{-13}$, but these results still compare favorably to earlier high-energy limits from LEP $\left(1.7 \cdot 10^{-6}\right)$ and $\operatorname{ATLAS}\left(7.5 \cdot 10^{-7}\right)$.

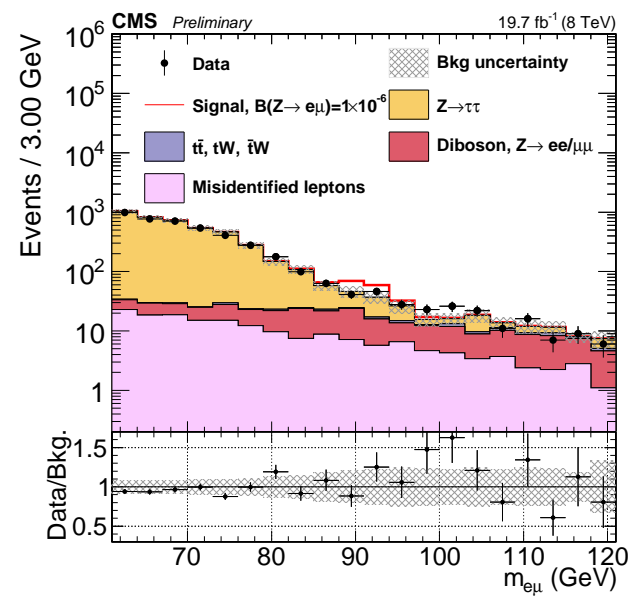

Figure 1: Data/simulation comparison plot of the lepton pair's invariant. The hypothetical $Z \rightarrow e \mu$ signal is included as a red line. No excess was found in data compared to the SM simulations [3].

\section{3. $\mathbf{H} \rightarrow \mathrm{LFV}$ search}

A CMS direct search of Higgs LFV decays on 2012 data $\left(19.7 \mathrm{fb}^{-1}, 8 \mathrm{TeV}\right)$ found a $2.4 \sigma$ excess in the $\mu \tau$ final state [4], which was not confirmed in the 2016 data analysis ( $35.9 \mathrm{fb}^{-1}$,

$13 \mathrm{TeV}$ ). The current best CMS 95\% CL observed limits on the branching ratios are: $B(\mathrm{H} \rightarrow \mathrm{e} \mu)<0.035 \%$ (from 2012 data), $B(\mathrm{Z} \rightarrow \mathrm{e} \tau)<0.61 \%$ and $B(\mathrm{Z} \rightarrow \mu \tau)<0.25 \%$ (2016) [5]. 

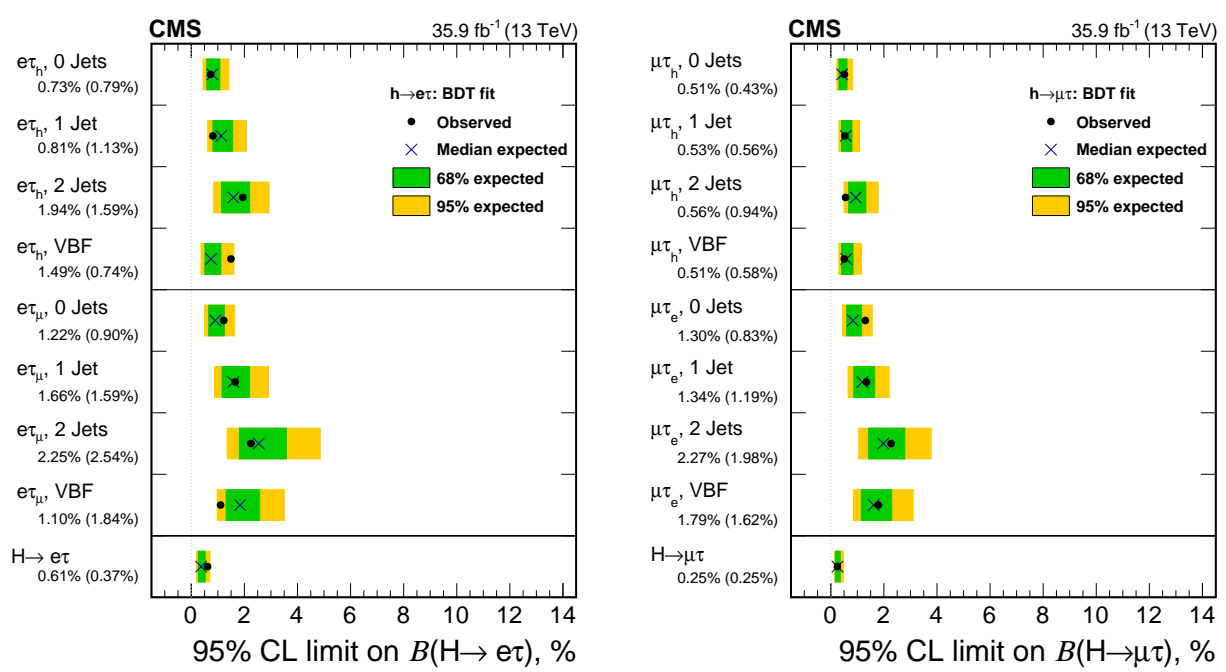

Figure 2: The two plots come from the 2016 analysis, and show the branching ration limits at the $95 \%$ confident level for the e $\mu$ and $\mu \tau$ final states. The excess in the $\mu \tau$ channel is gone, as the observed limit matches the expected limit [5].

\section{Heavy $X \rightarrow$ e $\mu$ search}

Direct searches for high mass LFV decays of new massive resonances have also been performed in the e $\mu$ channel. The latest such search was done on 2016 data [6]. 95\% CL exclusion limits have been set for RPV SUSY models, models with extra-dimensions, and for a Sequential Standard Model (SSM) Z'. In the RPV SUSY framework, a limit on the tau sneutrino mass of $m_{\tilde{v}_{\tau}}>1.7$ (resp. 3.8) TeV was found for couplings of 0.01 (resp. 0.1). For ADD models with 4-6 extra dimensions, the limits on the quantum black hole threshold mass were $m_{t h}>5.3-5.6 \mathrm{TeV}$. In a warped RS model, the limit was $m_{t h}>3.6 \mathrm{TeV}$. For the SSM, the limit on the Z' mass was $m_{Z^{\prime}}>4.4 \mathrm{TeV}$. All limits improved by at least $1 \mathrm{TeV}$ the previous CMS results.
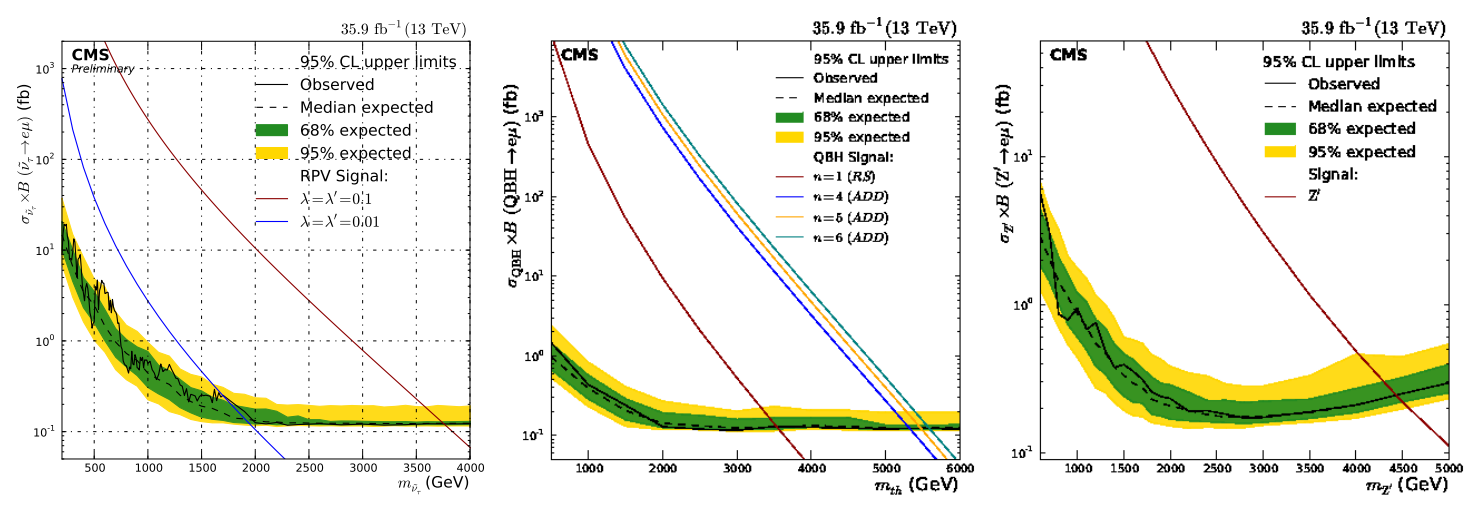

Figure 3: Limit contours on the (mass, cross section times branching ratio) plane. From left to right: RPV SUSY models, models with extra dimensions, SSM Z' [6]. 


\section{References}

[1] SuPER-KAmioKAnde Collaboration, Evidence for oscillation of atmospheric neutrinos, Phys. Rev. Lett. 81 (1998) 1562 [hep-ex/9807003].

[2] CMS Collaboration, The CMS Experiment at the CERN LHC, JINST 3 (2008) S08004.

[3] CMS Collaboration, Search for Lepton Flavor Violation in Z decays in pp collisions at sqrt $(s)=8 \mathrm{TeV}$, CMS-PAS-EXO-13-005 (2015) [https://cds.cern.ch/record/2019863? In=en].

[4] CMS Collaboration, Search for lepton flavour violating decays of the Higgs boson to e $\tau$ and e $\mu$ in proton-proton collisions at $\sqrt{s}=8 \mathrm{TeV}$, Phys. Lett. B763 (2016) 472 [1607.03561].

[5] CMS Collaboration, Search for lepton flavour violating decays of the Higgs boson to $\mu \tau$ and e $\tau$ in proton-proton collisions at $\sqrt{s}=13 \mathrm{TeV}, \mathrm{JHEP} 06$ (2018) 001 [1712.07173].

[6] CMS Collaboration, Search for lepton flavour violating decays of heavy resonances and quantum black holes to an e $\mu$ pair in proton-proton collisions at $\sqrt{s}=8 \mathrm{TeV}$, Eur. Phys. J. C76 (2016) 317 [1604.05239]. 